\title{
Regression assumptions in clinical psychology research practice - A systematic review of common misconceptions
}

\author{
Anja Franziska Ernst ${ }^{1}$, Casper J Albers ${ }^{\text {Corresp. } 1}$ \\ ${ }^{1}$ Heymans Institute for Psychological Research, University of Groningen, Groningen, The Netherlands \\ Corresponding Author: Casper J Albers \\ Email address: c.j.albers@rug.nl
}

Misconceptions about the assumptions behind the standard linear regression model are widespread and dangerous. These lead to using linear regression when inappropriate, and to employing alternative procedures with less statistical power when unnecessary. Our systematic literature review investigated employment and reporting of assumption checks in twelve clinical psychology journals. Findings indicate that normality of the variables themselves, rather than of the errors, was wrongfully held for a necessary assumption in $4 \%$ of papers that use regression. Furthermore, $92 \%$ of all papers using linear regression were unclear about their assumption checks, violating APA-recommendations. This paper appeals for a heightened awareness for and increased transparency in the reporting of statistical assumption checking. 
Regression Assumptions in Clinical Psychology Research Practice - A systematic review of common misconceptions

\author{
Anja F. Ernst and Casper J. Albers ${ }^{1)}$ \\ University of Groningen
}

Heymans Institute for Psychological Research, Groningen, The Netherlands. ${ }^{1)}$ Corresponding author: c.j.albers@rug.nl 
1

2

3 Regression Assumptions in Clinical Psychology Research Practice - A systematic review of common misconceptions

7 Misconceptions about the assumptions behind the standard linear regression model are

8 widespread and dangerous. These lead to using linear regression when inappropriate, and to

9 employing alternative procedures with less statistical power when unnecessary. Our systematic

10 literature review investigated employment and reporting of assumption checks in twelve clinical

11 psychology journals. Findings indicate that normality of the variables themselves, rather than of

12 the errors, was wrongfully held for a necessary assumption in $4 \%$ of papers that use regression.

13 Furthermore, $92 \%$ of all papers using linear regression were unclear about their assumption

14 checks, violating APA-recommendations. This paper appeals for a heightened awareness for and 15 increased transparency in the reporting of statistical assumption checking. 
18 Regression Assumptions in Research Practice - A systematic review of common misconceptions

19

One of the most frequently employed models to express the influence of several

predictors on a continuous outcome variable is the linear regression model:

$$
Y_{i}=\beta_{0}+\beta_{1} X_{1 i}+\beta_{2} X_{2 i}+\ldots+\beta_{p} X_{p i}+\varepsilon_{i}
$$

This equation predicts the value of a case $Y_{i}$ with values $X_{j i}$ on the independent variables $X_{j}(j=$ $1, \ldots, p$ ). The standard regression model takes $X_{j}$ to be measured without error (cf. Montgomery, Peck \& Vining, 2012, p.71). The various $\beta_{j}$ slopes are each a measure of association between the respective independent variable $X_{j}$ and the dependent variable $Y$. The error for the given $Y_{i}$, the difference between the observed value and value predicted by the population regression model, is denoted by $\varepsilon_{i}$ and is supposed to be unrelated to the values of $X_{p}$. Here, $\beta_{0}$ denotes the intercept, the expected $Y$ value when all predictors are equal to zero. The model includes $p$ predictor variables. In case $p=1$, the model is denoted as the simple linear regression model. The standard linear regression model is based on four assumptions. These postulate the properties that the variables should have in the population. The regression model only provides proper inference if the assumptions hold true (although the model is robust to mild violations of these assumptions). Many statistical textbooks (for instance, Miles \& Shevlin, 2001; Cohen, Cohen, West \& Aiken, 2003; Lomax \& Hahs-Vaughn, 2012; Montgomery et al., 2012) provide more background on these assumptions as well as advice on what to do when these assumptions are violated.

Violations of these assumptions can lead to various types of problematic situations. First, estimates may become biased, that is not estimating the true value on average. Second, estimators may become inconsistent, implying that convergence to the true value when the 
41 sample size increases is not guaranteed. Third, the ordinary least squares estimator may not be

42 efficient anymore: For instance, in the presence of assumption violations, OLS may provide less

43 accurate parameter estimates than other available estimation procedures. Fourth and finally,

44 NHST's and confidence intervals might become untrustworthy: $p$-values can be systematically

45 too small or too large, and confidence intervals are too narrow or too wide. This can occur even

46 if estimators are unbiased, consistent and efficient. For a more detailed description of these

47 issues, see Williams et al. (2013). Please note that these assumptions are the assumptions when

48 estimating using the Ordinary Least Squares (OLS) procedure, which is the default procedure in

49 many software packages, including SPSS and $R$. Other type of estimation methods, such as GLS,

50 apply other sets of assumptions.

$51 \quad$ Below, the four OLS-assumptions will be discussed.

52 Linearity. The conditional mean of the errors is assumed to be zero for any given

53 combination of values of the predictor variables. This implies that, for standard multiple

54 regression models, the relationship between every independent variable $X_{i}$ and the population

55 mean of the dependent variable $Y$, denoted by $\mu_{Y}$, is assumed to be linear when the other

56 variables are held constant. Furthermore, the relations between the various $X_{i}$ and $\mu_{Y}$ are additive:

57 thus, the relation of $X_{i}$ with $\mu_{Y}$ is the same, regardless of the value of $X_{j}(j \neq i)$. This relates to the

58 issue of multicollinearity; a good model is expected to have as little overlap between predictors

59 as possible. However, multicollinearity is not a model assumption but merely a necessity for a

60 model to be parsimonious. Violation of this assumption can obviously occur when non-linear

61 relations are unmodelled, but also in case of measurement error (see Williams et al., 2013).

$62 \quad$ Normality. All errors are normally distributed around zero. 
of the independent variables. Thus, this variance, which can then be denoted by a single symbol

65 (e.g. $\sigma^{2}$ ). This assumption is also called the homoscedasticity assumption. Thus, the second and

66

67 third regression assumptions combined specify that the errors $\left(\varepsilon_{i}\right)$ of the model should follow a normal distribution with a mean of zero and a (fixed) standard deviation $\sigma$. Heteroscedasticity often manifests itself through a larger spread of measurements around the regression line at one side of the scatterplot than at the other.

Independence. The errors $\varepsilon_{1}, \varepsilon_{2}, \ldots$, should be independent of one another: the pairwise covariances should be zero. This assumption is not directly based on the distribution of the data but on the study design and it requires the sampling method to be truly random (see, for instance, Cohen, Cohen, West and Aiken, 2003). As with the normality assumption, scatterplots alone are usually unsuitable for checking this assumption. A residual plot, or inspection of the autocorrelation of the residuals, is a better approach.

Common misconceptions about assumptions. There are many misconceptions about the regression model, most of which concern the assumptions of normality and homoscedasticity. Most commonly, researchers incorrectly assume that $X_{i}$, or both $X_{i}$ and $Y$, should be normally distributed, rather than the errors of the model. This mistake was even made in a widely-read article by Osborne and Waters (2002)attempting to educate about regression assumptions ${ }^{1}$ (cf. Williams, Grajales \& Kurkiewics, 2013).

Not assuming a normal distribution for $X_{i}$ may seem counterintuitive at first, however the indulgence of this assumption becomes more evident with an illustrative example. Take the standard Student's $t$-test which assesses if two distributions are statistically different from one

1 Based on the journal's access counter, there were more than 540,000 views at the time of this writing http://pareonline.net/genpare.asp? $w h=0 \& a b t=8$ 
85 another (e.g., a $t$-test that compares the efficacy of a specific treatment compared to a placebo

86 treatment). The population distributions in both conditions are assumed to be normally

87 distributed with equal variances. This $t$-test can also be expressed as a regression model where

88 the independent variable $X$ dummy codes the group membership, (i.e. if a participant is in the

89 control $=0$, or in the treatment condition, $X=1$ ). This regression model and the $t$-test are

90 mathematically equivalent and will thus lead to identical inference. Variable $X$ will only attain

91 two values, 0 and 1, as it is only used as label for group membership. The dependent variable $Y$

92 will attain many different values: following a normal distribution for the treatment group and a

93 (possibly other) normal distribution for the control group. This resulting 'condition membership'

94 distribution is nothing close to normal (as it takes on just two values), however no assumption of

95 the general linear model is violated because the subpopulations of $Y$ for each of the $X$ values

96 follow a normal distribution with equal variances, as is visualised in Figure 1. This example

97 demonstrates that the assumptions of the $t$-test (standard normal distribution of the populations

98 around the group mean and equal variances) coincide with the second regression assumption.

As a consequence of the second regression assumption, the distribution of the dependent variable conditional on some combination of values of the predictor variables is linear. Thus, $Y_{\mathrm{i}}$

102 is actually normally distributed around $\mu_{Y}$, the true conditional population mean. This becomes

103 clear when remembering that the error of the regression estimation is normally distributed

104 around mean zero and that $Y_{i}$ is equal to $\mu_{Y}+\varepsilon_{i}$. That is, individual observations are the sum of

105 the mean and a deviation from this mean. However, it is wrong to test the normality of the

106 marginal distribution of the dependent variable $Y$ because this would imply that all $\mu_{Y}$ values are 
107 the same which is, generally, not the case. (This situation occurs only when all regression slopes

108 are zero and, thus, all predictor variables are linearly unrelated to $Y$.)

109 Regarding the linearity assumption, a common misconception is in thinking that only

110 linear relationships can be modelled using the OLS framework. This is not the case: the linearity

111 assumption deals with linearity in the parameters and the estimates, but not necessarily in the

112 variables.

113 Consequences of violations of assumptions. Misconceptions like the ones outlined above

114 potentially have severe effects on the ability to draw inferences from a data analysis. First of all,

115 the checking of wrong assumptions will most likely lead to the neglect of correct assumption

116 checking. If the researcher will decide on a regression analysis without having tested the correct

117 assumptions it is possible that some requirements of linear regression were not met. However, in

118 any case the neglect of correct assumption checking will always leave the reader or reviewer

119 unable to trust the results because there is no way of knowing whether the model assumptions

120 were actually met. Of course, the severity of this problem of non-transparency persists even

121 when the researcher ensured the validity of all necessary assumptions and merely failed to report

122 those findings. Not only does such non-transparency in data analysis lead to confusion for

123 researchers that are potentially interested in replicating or comparing the results, it also weakens

124 the informational value of the research findings that are being interpreted.

125 A second problem that is caused by misconceptions about model assumptions occurs

126 when a researcher decides against a linear regression analysis because of the violation of faulty

127 assumptions that were unnecessary in the first place. The difficulty of abandoning linear

128 regression analysis for a non-parametric procedure is the fact that the ordinary least squares

129 method of linear regression is a more powerful procedure than any of its non-parametric 
130 counterparts, if its assumptions are met. Hence, wrongfully deciding against the employment of

131 linear regression in a data analysis will lead to a decrease in power. Thus, the understanding of

132 the correct regression assumptions is crucial because it prevents the abandonment of the linear

133 regression technique in cases in which it would be unjustified. Furthermore, the checking of

134 assumptions has another advantage: it might help the researcher to think about conceptually

135 alternative models. For instance, heteroscedasticity in the data could be a sign of an interaction

136 between one or more of the included independent variables and an independent variable not (yet)

137 included in the model.

138 Applying a linear regression model when assumptions are violated can lead to (severe)

139 problems, but this does not have to be the case, depending on the type of violation. Violations of

140 the linearity assumption and of the independence assumption can lead to biased, inconsistent and

141 inefficient estimates (Chatterjee \& Hadi, 2006; Williams et al., 2013). A proper check on these

142 two assumptions is thus vital. The consequences of violations are less severe for the other two

143 assumptions.

144 If normality of errors holds, the OLS method is the most efficient unbiased estimation

145 procedure (White \& MacDonald, 1980). If this assumption does not hold (but the remaining

146 assumptions do), OLS is only most efficient in the class of linear estimators (see Williams et al.,

147 2013, for a detailed discussion). This implies that, as long as the other assumptions are met,

148 estimates will still be unbiased and consistent in the presence of a normality violation, but the $p$ -

149 values might be biased. Furthermore, the central limit theorem implies that for large samples the

150 sampling distribution of the parameters will be at least approximately normal, even if the

151 distribution of the errors is not. Hence, the regression model is robust with respect to violations 
152 of the normality assumption. Potential problems will, in practice, primarily occur for inferential

153 problems (such as confidence intervals and testing) with small samples.

154 Similarly, violations of the homoscedasticity assumption are not necessarily problematic.

155 Provided that the very mild assumption of finite variance holds, estimates will still be unbiased

156 and consistent (Chatterjee \& Hadi, 2006).

157 Best practices for checking of assumptions. There are many different ways to check the

158 four assumptions of the regression model and there generally is no 'uniformly optimal' approach.

159 Generally, there are two classes of approaches: (i) formal tests (of the style ' $\mathrm{H}_{0}$ : the

160 assumption is true' vs ' $\mathrm{H}_{\mathrm{A}}$ : the assumption is violated') and (ii) graphical methods. For the

161 normality assumption alone, there is an abundance of formal tests, such as the Shapiro-Wilk test,

162 the Anderson-Darling test and the Kolmogorov-Smirnov test. Which approach is most powerful

163 depends on the kind of violation from normality (Razali \& Wah, 2011). However, the use of

164 formal tests is discouraged by some (Albers, Boon \& Kallenberg, 2000, 2001). When the

165 normality assumption holds, the null hypothesis of normality will still be rejected in $\alpha$ (usually

$1665 \%$ ) of cases. This distorts the $p$-value distribution of the estimates of the regression model, even

167 when no assumptions are violated. Furthermore, tests for normality only have adequate power in

168 case of large sample sizes. However, when the sample size is large, the central limit theorem

169 implies that violations of normality have only limited effect on the accuracy of the estimates.

170 Applying graphical methods is therefore a preferred approach. This is also suggested by

171 the statistical guidelines for the APA set up by Wilkinson et al. (1999, p. 598): "Do not use

172 distributional tests and statistical indices of shape (e.g. skewness, kurtosis) as a substitute for

173 examining your residuals graphically". This advice builds upon the adagium by Chambers et al.

174 (1983, p. 1) that "there is no single statistical tool that is as powerful as a well-chosen graph". A 
175 graph simply provides more information on an assumption than a single $p$-value ever can (see

176 also Chatterjee \& Hadi, 2006, Ch. 4).

177 The linearity assumption can easily be checked using scatterplots or residual plots: plots

178 of the residuals vs. either the predicted values of the dependent variable or against (one of) the

179 independent variable(s). Note that residuals are the differences between the observed values and

180 the values predicted by the sample regression model, whereas errors denote the difference with

181 the values predicted by the population regression model. Residual plots are also the best visual

182 check for homoscedasticity. For the normality assumption, it is difficult to judge on the basis of a

183 scatterplot whether the assumption is violated. A histogram of the residuals is also a poor visual

184 check, as the 'shape' of the histogram heavily depends on the arbitrary choice of the bin width,

185 especially in small samples. Normal probability plots, or QQ-plots, provide a much better way to

186 check normality. Finally, a check on the independence assumption is done by studying the

187 autocorrelation function of the residuals. Note that this latter check does check for temporal

188 dependence violations of the independence assumptions, but not for other possible violations

189 such as clustering of observations. Furthermore, a common violation of independence involves

190 repeated-measures designs in which each individual contributes a set of correlated responses to

191 the data because of individual differences.

192

Outline of this paper. Misconceptions about frequently employed statistical tools, like

194 the $p$-value, are not rare, even amongst researchers (see Bakker and Wicherts, 2011; Hoekstra,

195 Morey, Rouder \& Wagenmakers, 2014). Our paper aims to shed light onto potential

196 misconceptions researchers and reviewers might hold about the linear regression model.

197 Therefore, the documentary practices of psychological research papers with the linear regression 
198 model and its assumptions were investigated by means of a literature review. In this review, we

199 investigate the proportion of papers where misconceptions around the assumptions of the

200 statistical regression model occurred and which type of misconceptions occurred most often.

201 This will provide important information, as the first step in solving flawed methodology in

202 research is finding out where the flaws are and how predominant they are.

203 Although the consequences of incorrectly dealing with assumptions can be severe, the

204 APA manual (American Psychological Association, 2010) barely provides guidelines on what to

205 report and how to report. It does recommend being specific about "information concerning

206 problems with statistical assumptions and/or data distributions that could affect the validity of

207 findings" (p. 248) as part of the Journal Article Reporting Standards, but this is not obligatory.

208 The APA Task Force on Statistical Inference (Wilkinson and Task Force on Statistical Inference,

209 1999) is more explicit in their recommendations: "You should take efforts to assure that the

210 underlying assumptions required for the analysis are reasonable given the data. Examine the

211 residuals carefully.” (p. 598).

212 In this manuscript we present the findings of our literature review. Because the whole field of

213 psychological science is too broad to study in a single paper, we restrict ourselves to the field of

214 clinical psychology. We investigate how statistical assumptions were covered in various journals

215 of clinical psychology and what types of misconceptions and mistakes are occurring most often.

216 In the discussion section, possible explanations for the reported findings will be offered. The

217 paper will conclude with several proposals of how potential shortcomings in the current practices

218 with linear regression analysis could be overcome. 
220 Journals. The literature review restricted itself to articles that were published in clinical

221 psychology journals in the year 2013. It is possible that problems with the checking of

222 assumptions are less (or more) prominent in journals with a high impact, which is why we aimed

223 for a selection of journals with varied impact factors. We employed the Scientific Journal

224 Rankings (SJR) as reported on 16 December 2014 by the SCImago Journal and Country Rank

225 (SCImago, 2007) for clinical psychology journals of the year 2013 to divide all clinical

226 psychology journals into four quartiles $(\mathrm{Q} 1-\mathrm{Q} 4)$, where Q1 contains the $25 \%$ of journals with

227 the highest journal rank, etcetera. From every quartile the three highest ranked journals were

228 selected to be included in the review. Hence, we obtained a balanced selection from all clinical

229 psychology journals, as listed in Table 1. All articles published in the selected journals in 2013

230 were included, including also papers that had potentially been published online earlier. Letters,

231 journal corrigenda, editorial board articles and book reviews were not included in the review.

232 Basically, articles that were by design not containing a method section were not included in our

233 lists of articles. The focus of this review purely lies on published scientific articles.

234 Every article was retrieved directly from the official website of its respective journal

235 (except for Q1.3 which was directly retrieved from its official database "PsycARTICLES"). All

236 articles were in German (Q3.1), Spanish (part of Q3.3) or in English (all other). German articles

237 were also included in the review; Spanish articles were excluded because of the authors' lack of

238 proficiency in this language. Figure 2 displays the Prisma workflow of the analysis. We

239 conducted our review adhering to common meta-regression guidelines (Moher, Liberati,

240 Tetzlaff, Altman, The PRISMA Group, 2009).

241 Procedure. We evaluated whether and how papers described careful examination of the data with

242 regard to the underlying model assumptions whenever conducting statistical analysis (APA, 
243 2010; Wilkinson et al., 1999). Papers were skimmed for the following criteria: if they had used

244 linear regression, how they tested the regression assumptions or what kind of assumptions they

245 indicated as being necessary, if they had transformed data on basis of correct or incorrect

246 assumptions and if a paper had considered an ordinary least squares regression model but

247 employed a different model on basis of either correct or incorrect assumptions. This resulted in a

248 classification scheme of 12 different rubrics which are displayed in Table 2. This scheme is

249 mutually exclusive and exhaustive; all studied papers are classified into exactly one rubric. The

250 search strategy has been carried out by Anja Ernst. Independently, Casper Albers checked and

251 classified 10\% of the manuscripts in the Q1-journals. No mismatch between both sets of

252 classifications occurred.

253 Papers that used linear regression were classified as follows. We assumed the most

254 common misconception about linear regression to be the checking of the normality of the

255 variables while failing to check the normality of the errors. Therefore, we created rubrics 8 to 11

256 to classify all papers that employed linear regression and checked or assumed the normality of $X$

257 and/or $Y$ but not of the errors. An example of a paper classified in rubric 8 stated "Variable

258 distributions were tested to ensure assumptions of normality, linearity, and variance equality

259 were met, with no significant violations observed" (Nadeau, Lewin, Arnold, Crawford, Murphy

$260 \&$ Storch, 2013). Often, when the normality assumption was mentioned it was unclear whether

261 authors had checked the normality of errors or of the variables. Articles that were unclear in this

262 regard were classified under rubric 5. For instance, one of the articles classified in this rubric

263 stated "Preliminary analysis examined data for the presence of outliers and the appropriateness

264 of assumptions of normality, linearity, and homoscedasticity" (Nguyen, Barrash, Koenigs,

265 Bechara, Tranel \& Denburg, 2013) with no more information provided on the assumption 
266 checks. Papers that indicated to have checked the homoscedasticity, normality of the errors and

267 linearity assumptions were classified as 'Correct' in rubric 4. Articles that mentioned at least a

268 few correct assumptions, as opposed to giving no indication at all (rubric 7), were classified in

269 rubric 6. Because all papers that checked or assumed the normality of $X$ or $Y$ but not of the errors

270 were included in rubrics 8 to 11 , we have named rubric 6 'Did not test all but some correct

271 assumptions, did not include normality of variables'. After performing the literature review it

272 became apparent that none of the articles listed in this category had mentioned the normality of

273 errors. Because we aimed to demonstrate how rare it is to read that researchers check the

274 normality of the errors we have updated the name of the category into 'Did not test all but some

275 correct assumptions, included neither normality of variables nor errors', even though the

276 checking of the normality of errors was not employed as a criterion for inclusion in this category

277 during the literature review.

278 Papers that did not fit into any of the eleven other rubrics but included an aspect on linear

279 regression assumptions that we found unsatisfactory were listed in the rubric 'Other

280 misconceptions about assumptions'. One example of a paper classified in this category claimed

281 "All assumptions of multiple regression (linearity, multicollinearity, and homoscedasticity) were

282 met" this paper was included in the category 'Other misconceptions' because they did not only

283 lack any mention whether normality of the residuals was checked (which would have resulted in

284 a classification in rubric 6) but also claimed that a list not containing normality of residuals was

285 complete. We found this claim unsatisfactory which was the reason we included this paper in

286 rubric 12.

287 Whenever an article in our selection reported the results of a regression analysis of

288 another paper or reviewed several linear regression articles, it was evaluated whether the paper 
289 reviewing all the previous regression analysis had made it a criterion of inclusion whether the

290 assumptions have been met in the original articles. If a review article did not check or mention

291 the assumptions of the papers that published the original analysis, the article was classified as

292 'Use of linear regression but no indication if any or which assumptions were tested'. However,

293 these sorts of papers constitute less than one percent of our selected articles. It should be noted

294 that this only applies to papers which reported the data values of a linear regression or analysed

295 regression results from other studies. A paper was not included if it only mentioned the direction

296 of the outcomes of another paper's regression model or stated that a relationship had been

297 established by previous research findings.

298 Because the focus of this paper lies on the assumptions of linear regression, only linear

299 regression model assumptions were examined in the literature review. Consequently, papers that

300 analysed data by means of other types of regression, such as latent factor models, logistic

301 regression, and proportional hazards models (Cox regression), were not inspected for assumption

302 checking. When a paper used a regression model other than linear regression, and without

303 mentioning that linear regression was alternatively considered for data analysis it was classified

304 as 'No Model of Interest'. 
307 display the number of occurrences of different classifications for the selected journals. In the

308 online supplementary material we indicate for all of the 893 individual papers studied into which

309 category they fall.

310 Table 3 shows the findings for all journals with the 12 different classification rubrics

311 summarized into seven different columns. The three columns entitled 'Dealing with

312 assumptions' list the number of different types of regression papers in a specific journal and

313 shows the proportional amount of this type in relation to the complete number of regression

314 articles in that journal. The two columns for 'No regression' list the number of papers which did

315 not use a linear regression model and included in their method sections to have considered a

316 linear regression analysis but decided against it on the basis of checking either correct or

317 incorrect assumptions.

318 Table 4 specifies the details behind the articles which are listed in Table 3 under the

319 column titled 'incorrectly'. This table classifies the corresponding 10 papers into Rubrics $8-12$

320 of Table 2 . It may be noted that $4 \%$ of all articles that used linear regression checked normal

321 distributions of some variables instead of normal distribution of errors.

322 Table 5 specifies the details behind the column 'unclear' in Table 2; that is it classifies

323 the 159 corresponding papers into Rubrics 5 to 7 of Table 2. Of all papers that employed

324 regression, 92\% were unclear about the assumptions of the linear regression model that were

325 tested or were thought to be fulfilled. 


\section{Discussion}

In our analysis, we studied 893 papers, representative for the work published in the field

328 of clinical psychology, and classified the 172 papers (19.4\%) which considered linear regression

329 into three categories: those that dealt with the assumptions correctly, those that dealt with

330 assumptions incorrectly, and those that did not specify how they dealt with assumptions.

331 Merely $2 \%$ of these papers were both transparent and correct in their dealing with

332 statistical assumptions. Furthermore, in $6 \%$ of papers transparency was given but the dealing

333 with assumptions was incorrect. Hoekstra, Kiers \& Johnson (2012) might provide some insight

334 into why researchers did not check assumptions. They list unfamiliarity with either the fact that

335 the model rests on the assumption or with how to check the assumption as the top two reasons.

336 As explained, incorrect dealing with the assumptions could lead to severe problems regarding the

337 validity and power of the results. We hope that this manuscript creates new awareness of this

338 issue with editors of clinical psychology journals and that this will assist in bringing down the

339 number of publications with flawed statistical analyses.

340 A tremendous amount of papers that employed regression, $92 \%$ of those studied, were

341 not clear on how they dealt with assumptions. It is not possible (not for us, nor for the reader) to

342 judge from the text whether checks for assumption violations were performed correctly. In the

343 group of transparent papers, the number of papers with fundamental mistakes in dealing with

344 assumptions far outnumber the number of papers without mistakes. Thus, even though it is not

345 possible to pinpoint an exact number to it, it would be naive to assume that only a small

346 proportion of those $92 \%$ also deal with assumptions incorrectly.

347 We believe that most contemporary problems in the handling of regression methods

348 could be counteracted by a more thorough coverage of the statistical assumption checks that 
349 were performed in order to determine the validity of the linear regression model. At the very

350 least, transparency regarding how assumptions are approached, in line with the recommendations

351 by Wilkinson et al. (1999), is essential. Thus, mentioning which assumptions were checked and

352 what diagnostic tools were used to check them under what criteria, should be a minimum

353 requirement. Preferably, the authors should also show the results of these checks.

354 With transparency, the critical reader can distinguish correct approaches from incorrect

355 ones, even if the author(s), editor(s) and referees fail to spot the flaws. These statistical checks

356 can be given in the paper itself, but could also be provided in online supplementary material, a

357 possibility most journals offer nowadays (note that none of the papers investigated in this

358 manuscript referred to supplementary material for assumption checks). Thus, increased length of

359 the manuscript does not need to be an issue. Our aspiration for an increased transparency in

360 statistical assumption checks is in line with recent developments in psychology such as open

361 methods (obligatory in e.g. the APA-journal Archives of Scientific Psychology) and open data

362 (either published as online supplementary material with a paper, or through special journals like

363 Journal of Open Psychology Data). With open data, sceptical scientists can re-do the analyses

364 and check assumptions for themselves. Enforcing, or at least strongly encouraging, transparency

365 can even have beneficial effects to the level of publications in the respective journal (Wicherts,

366 Bakker \& Molenaar, 2011). Even if publishing the data does not have a direct beneficial effect

367 on the quality of work, it will be useful as it provides the sceptical reader with the required

368 information to perform the assumption checks and thus the possibility to check the credibility of

369 the published work. 
372 adequately than lower ranking journals. Even though the results in Table 5 indicate that higher

373 ranked journals were more likely to test at least a few assumptions compared to lower ranked

374 journals; the results do mainly show that there is great variability between journals regarding the

375 number of papers with applied regression models they publish: two journals published no papers

376 in 2013 that employed linear regression, and five journals published six or fewer of these papers.

377 Because two of the three inspected Q1 journals are review journals they predominantly

378 employed meta-regression, a special type of regression useful for conducting meta-analyses, and

379 only rarely linear regression, it should be pointed out that of the 15 papers that used meta-

380 regressions in our Q1.2 eleven tested at least some of the required assumptions (that is $73 \%$ of

381 meta-regression papers were checked correctly for statistical assumptions). We believe that for

382 these papers the percentage is much better than the overall percentage of $2 \%$ for applied

383 regression papers, because meta-analyses are usually carried out by a team of authors including

384 at least one statistician or psychometrician.

385 We have limited our literature review to papers employing linear regression models, in

386 order to keep the study feasible. We suspect that similar findings would arise when studying

387 other classes of statistical models. Furthermore, we have also limited the review to papers

388 published in the field of clinical psychology; however we suspect that similar problems occur-

389 albeit possibly in different proportions - in all areas of applied psychological research. Thus, our

390 suggestions with respect to increased transparency and better evaluation of the employed

391 methodology should be relevant for a wider range of papers than those studied here. Because our

392 categorization of papers is reasonably straightforward, only one author conducted most of the

393 review. While our rubrics allow objective classifications we cannot preclude a few single 
394 accidental misclassifications. However, possible misclassification should be minimal at most and

395 can therefore be expected to not have skewed the overall results that are based on a large number

396 of papers. Thus, despite this limitation we are confident in the overall results. For future

397 research, it would be interesting to do a similar literature review based on either alternative

398 techniques or on another field of application. Furthermore, more research is needed in

399 understanding the reasons that underlie why researchers frequently do not check assumptions.

400 One of the consequences of the lack of reporting of assumption checks is that many

401 published findings in clinical psychology are underestimating the uncertainty in their claims. For

402 instance, reported confidence intervals in the literature describe the uncertainty surrounding the

403 parameter, if the OLS-assumptions are met. The uncertainty of the validity of the assumptions

404 should lead to wider confidence intervals, in general. For future research, it would be an

405 interesting puzzle to assess the magnitude of this added uncertainty.

406 To summarise, in order to prevent the observed problems that were outlined above we

407 suggest a more transparent methodological reporting. Research should cover which assumption

408 checks were carried out. Furthermore, it should be mentioned if alternative statistical models

409 have been considered and why they were not employed, if so. This will be a necessity for future

410 research articles in order to be able to detect and prevent errors related to widespread

411 misconceptions but also to remove doubt from articles with an actual immaculate data analysis.

413

\section{Additional information}

415 A detailed breakdown of the systematic review, references to all websites employed to retrieve

416 articles as well as a completed PRISMA checklist are provided as online supplementary material. 


\section{References}

419

420 Albers, W., Boon, P. C., \& Kallenberg, W. C. M. (2000). Size and power of pretest procedures. 421 Annals of Statistics, 28, 195-214. Retrieved from: http://www.jstor.org/stable/2673986.

422

423

Albers, W., Boon, P. C., \& Kallenberg, W. C. M. (2001). Power gain by pre-testing? Statistics \& Decisions, 19(3), 254-276.

424 American Psychological Association (2010). Publication Manual of the American

Psychological Association (Sixth Edition). Washington D.C.: American Psychological 426

Association.

427

428

429

430

431

432

433 434

435

436

437

438

Bakker, M. \& Wicherts, J. M. (2011). The (mis)reporting of statistical results in psychology journals. Behavior Research Methods, 43, 666-678, doi:10.3758/s13428-011-0089-5

Chambers, J. M., Cleveland, W. S., Kleiner, B., Tukey, P. A. (1983). Graphical Methods for Data Analysis. Pacific Grove, CA: Wadsworth \& Brooks/Cole

Cohen, J., Cohen, P., West, S.G., Aiken, L.S. (2003). Applied Multiple Regression/Correlation Analysis for the Behavioral Sciences (Third Edition). New York, NY: Routledge.

Chatterjee, S. \& Hadi, A. S. (2006). Regression Analysis by Example, Fourth Edition. Hoboken, NJ:

John Wiley \& Sons.

Hoekstra, R., Kiers, H. A. L., \& Johnson, A. L. (2012). Are assumptions of well-known statistical techniques checked, and why (not)? Frontiers in Psychology, 3, 137, doi:10.3389/fpsych.2012.00137 
439 Hoekstra, R., Morey, R. D., Rouder, J. N., \& Wagenmakers, E. J. (2014). Robust

440 misinterpretation of confidence intervals. Psychonomic Bulletin \& Review, 21, 1157-

441 1164. doi:10.3758/s13423-013-0572-3/21.12/2014

442 Lomax, R.G., \& Hahs-Vaughn, D. L. (2012). Statistical Concepts: A Second Course. New York, $443 \quad$ NY: Routhledge.

444 Miles, J. \& Shevlin, M. (2001). Applying Regression and Correlation: A Guide for Students and 445 Researchers. London, UK: Sage.

446 Moher, D., Liberati, A., Tetzlaff, J., Altman, D.G., The PRISMA Group (2009). Preferred 447 Reporting Items for Systematic Reviews and Meta-Analyses: The PRISMA Statement. 448 PLoS Medicine 6(6): e1000097. doi:10.1371/journal.pmed1000097/21.12/2014

449 Montgomery, D. C., Peck, E. A., \& Vining, G. G. (2012). Introduction to Linear Regression $450 \quad$ Analysis ( $5^{\text {th }}$ edition). New York: John Wiley \& Sons.

451 Nadeau, J. M., Lewin, A. B., Arnold, E. B., Crawford, E. A., Murphy, T. K., \& Storch, E. A. 452 (2013). Clinical correlates of functional impairment in children and adolescents with 453 obsessive-compulsive disorder. Journal Of Obsessive-Compulsive And Related Disorders, 2(4), 432-436. doi:10.1016/j.jocrd.2013.10.002

Nguyen, C. M., Barrash, J., Koenigs, A. L., Bechara, A., Tranel, D., \& Denburg, N. L. (2013).

Osborne, J. \& Waters, E. (2002). Four assumptions of multiple regression that researchers should always test. Practical Assessment, Research \& Evaluation, 8(2), 1-9. Retrievedfrom: http://PAREonline.net/getvn.asp?v=8\&n=2/16.12/2014 
462 Razali, N. \& Wah, Y. B. (2011). Power comparisons of Shapiro-Wilk, Kolmogorov-Smirnov, 463 Lilliefors and Anderson-Darling tests. Journal of Statistical Modeling and Analytics, $464 \quad 2(1): 21-33$.

465 SCImago. (2007). SJR - SCImago Journal \& Country Rank. http://www.scimagojr.com.

466 Retrieved

467

on: 16.12 .2014 .

468 Tabachnick, B. G., \& Fidell, L. S. (2013). Using multivariate statistics, $6^{\text {th }}$ edition. London, UK: 469 Pearson.

470 Wicherts, J. M., Bakker, M. \& Molenaar, D. (2011). Willingness to share research data is related 471 to the strength of the evidence and the quality of reporting of statistical results. PLOS

472 One, 6(11), doi: 10.1371/journal.pone.0026828

White, H. \& MacDonald, G. M. (1980). Some large-sample tests for nonnormality in the linear regression model. Journal of the American Statistical Association, 75(369): 16-28, doi:10.2307/1912934.

Wilkinson, L. \& Task Force on Statistical Inference (1999). Statistical Methods in Psychology Journals: Guidelines and Explanations. American Psychologist, Vol. 54, No. 8, 594-604 regression: Correcting two misconceptions. Practical Assessment, Research \& Evaluation, 18(11), 1-14. 
Tables

\begin{tabular}{ll}
\hline Label & Journal \\
\hline Q1.1 & Annual Review of Clinical Psychology \\
\hline Q1.2 & Clinical Psychology Review \\
\hline Q1.3 & Journal of Consulting and Clinical Psychology \\
\hline Q2.1 & International Psychogeriatrics \\
\hline Q2.2 & Journal of Attention Disorders \\
\hline Q2.3 & American Journal of Drug and Alcohol Abuse \\
\hline Q3.1 & Zeitschrift fur Klinische Psychologie und Psychotherapie \\
\hline Q3.3 & Journal of Obsessive-Compulsive and Related Disorders \\
\hline Q4.1 & International Journal of Psychology and Psychological Therapy \\
\hline Q4.2 & Internet Journal of Mental Health \\
\hline Q4.3 & Indian Journal of Psychological Medicine \\
\hline Table 1: Selection of Clinical Psychology Journals. The first column gives the ranking of the
\end{tabular}

483 Table 1: Selection of Clinical Psychology Journals. The first column gives the ranking of the

484 journal, the first number denoting the quartile in which the journal falls, the second number the

485 rank of the journal within that quartile.

486

\begin{aligned} & \hline \multicolumn{1}{l}{ Class. } Reason \\ & \hline Papers without a linear regression model: \\ & \hline 1 No Model of Interest \\ & \hline 2 Rejection of linear regression on basis of correct assumptions \\ & \hline 3 Rejection of linear regression on basis of not meeting incorrect assumptions \\ & \hline Papers with a linear regression model: \\ & \hline 4 Correct linear regression \\ & \hline 5 Mentioned all correct assumptions but not if the 'normality assumption' was tested on the residuals or on \\ &$X$ or $Y \\ &$\hline 6 Did not test all but some correct assumptions, included neither normality of variables nor errors \\ & \hline 7 Use of linear regression but no indication if any or which assumptions were tested \\ & \hline 8 Assumed/tested normally distributed $X$ but not the normality of the errors \\ & \hline 9 Assumed/tested normally distributed $Y$ but not the normality of the errors \\ & \hline 10 Assumed/tested normally distributed $X$ and $Y$ but not the normality of the errors \\ & \hline 11 Assumed/tested normally distributed variables but did not indicate if $X$ or $Y$ or both and did not test the \\ & normality of the errors \\ & \hline 12 Other misconceptions about assumptions \\ & \hline\end{aligned}

487

488 Table 2: Classification of the reviewed regression papers. Rubrics 3 and $5-12$ represent papers

489 with imperfect handling of regression assumptions: in rubrics $5-7$ it is unclear from whether

490 assumptions are correctly dealt with; in rubrics $8-12$ the dealing with assumptions was

491 incorrect. 


\begin{tabular}{|c|c|c|c|c|c|c|c|}
\hline \multirow[t]{2}{*}{ Journal } & \multirow{2}{*}{$\begin{array}{l}\text { Number } \\
\text { of papers } \\
\text { (rub. } 1- \\
12 \text { ) }\end{array}$} & \multirow[b]{2}{*}{$\begin{array}{l}\text { Number of } \\
\text { papers } \\
\text { with } \\
\text { regression } \\
\text { (rub. 4-12) }\end{array}$} & \multicolumn{3}{|c|}{ Dealing with assumptions } & \multicolumn{2}{|c|}{ No regression } \\
\hline & & & $\begin{array}{c}\text { Correctly } \\
(r u b .4)\end{array}$ & $\begin{array}{c}\text { Unclear } \\
\text { (rub. 5-7) }\end{array}$ & $\begin{array}{c}\text { Incorrectly } \\
(r u b .8- \\
12)\end{array}$ & $\begin{array}{l}\text { Correct } \\
\text { (violation of } \\
\text { true assump- } \\
\text { tion) }(r u b .2 \text { ) }\end{array}$ & $\begin{array}{l}\text { Incorrect } \\
\text { (violation of } \\
\text { false assump- } \\
\text { tion) }(r u b .3 \text { ) }\end{array}$ \\
\hline Q1.1 & 33 & 0 & 0 & 0 & 0 & 0 & 0 \\
\hline Q1.2 & 86 & $6(7 \%)$ & 0 & $6(100 \%)$ & 0 & 0 & 0 \\
\hline Q1.3 & 98 & $26(28 \%)$ & 0 & $25(100 \%)$ & 0 & $3(100 \%)$ & $\overline{0}$ \\
\hline Q2.1 & 227 & $44(19 \%)$ & $3(7 \%)$ & $39(89 \%)$ & $2(5 \%)$ & $1(100 \%)$ & 0 \\
\hline Q2.2 & 199 & $52(26 \%)$ & 0 & $49(94 \%)$ & $3(6 \%)$ & 0 & 0 \\
\hline Q2.3 & 54 & $14(26 \%)$ & 0 & $14(100 \%)$ & 0 & 0 & 0 \\
\hline Q3.1 & 23 & $5(22 \%)$ & 0 & $5(100 \%)$ & 0 & $1(50 \%)$ & $1(50 \%)$ \\
\hline Q3.2 & 59 & $21(55 \%)$ & 0 & $16(71 \%)$ & $5(29 \%)$ & $1(100 \%)$ & 0 \\
\hline Q3.3* & $10^{*}$ & $2(20 \%)^{*}$ & $0 *$ & $2(100 \%)^{*}$ & $0^{*}$ & $0^{*}$ & $0^{*}$ \\
\hline Q4.1 & 2 & $1(50 \%)$ & 0 & $1(100 \%)$ & 0 & 0 & 0 \\
\hline $\mathrm{Q} 4.2$ & 82 & 0 & 0 & 0 & 0 & 0 & 0 \\
\hline Q4.3 & 20 & $2(10 \%)$ & 0 & $2(100 \%)$ & 0 & 0 & 0 \\
\hline Total & 893 & $172(19 \%)$ & $3(2 \%)$ & $159(92 \%)$ & $10(6 \%)$ & $6(86 \%)$ & $1(14 \%)$ \\
\hline
\end{tabular}

493

494 Table 3: Proportion of various types of papers in our selected journals. Categorisations are mutually

495 exclusive and exhaustive. Journals are referred by the labels assigned in Table 1. "Rub." refers to the

496 rubrics in Table 2. The online supplementary material indicates which papers belong to each of the

497 numbers in this table.

498

* Papers in Spanish excluded

499

500

\begin{tabular}{|c|c|c|c|c|c|c|}
\hline Journal & $\begin{array}{l}\text { Articles with } \\
\text { flawed linear } \\
\text { regression } \\
\text { model (rub. } \\
\text { 8-12) }\end{array}$ & $\begin{array}{l}\text { Tested } \\
\text { normality } \\
\text { of X but not } \\
\text { of residuals } \\
\text { (rub. 8) }\end{array}$ & $\begin{array}{l}\text { Tested } \\
\text { normality } \\
\text { of Y but not } \\
\text { of } \\
\text { residuals } \\
\text { (rub. 9) } \\
\end{array}$ & $\begin{array}{l}\text { Assuming normally } \\
\text { distributed variables } \\
\text { but did not indicate } \\
\text { if X or Y or both } \\
\text { (rub.10) }\end{array}$ & $\begin{array}{l}\text { Tested } \\
\text { normality of } X \\
\text { and of Y but } \\
\text { not of } \\
\text { residuals (rub. } \\
\text { 11) } \\
\end{array}$ & $\begin{array}{l}\text { Other } \\
\text { misconceptions } \\
\text { (rub. 12) }\end{array}$ \\
\hline Q2.1 & 2 & 0 & 0 & & 0 & $2(100 \%)$ \\
\hline Q2.2 & 3 & $2(67 \%)$ & 0 & 0 & 0 & $1(33 \%)$ \\
\hline Q3.2 & 5 & $4(80 \%)$ & $1(20 \%)$ & 0 & 0 & 0 \\
\hline Total & 10 & $6(60 \%)$ & $1(10 \%)$ & 0 & 0 & $3(30 \%)$ \\
\hline
\end{tabular}


502 Table 4: Breakdown of the types of mistakes that were observed. Only Journals with flawed

503 models are listed. Categorizations are mutually exclusive and exhaustive. Journals are referred

504 by the labels assigned in Table 1.

505

\begin{tabular}{|c|c|c|c|c|}
\hline \multirow[t]{2}{*}{ Journal } & \multirow{2}{*}{$\begin{array}{l}\text { Papers in which } \\
\text { handling of regression } \\
\text { assumption was unclear } \\
(\text { rub. 5-7) }\end{array}$} & \multicolumn{3}{|c|}{ Unclear } \\
\hline & & $\begin{array}{l}\text { if the 'normality assumption' } \\
\text { was tested on the residuals or } \\
\text { on } X \text { or } Y(r u b .5)\end{array}$ & $\begin{array}{l}\text { Did not test all but } \\
\text { some correct } \\
\text { assumptions (rub. 6) }\end{array}$ & $\begin{array}{l}\text { no indication if any or } \\
\text { which assumptions } \\
\text { were tested (rub. 7) }\end{array}$ \\
\hline Q1.2 & 6 & 0 & $2(33 \%)$ & $4(67 \%)$ \\
\hline Q1.3 & 26 & 0 & 0 & $25(100 \%)$ \\
\hline Q2.1 & 39 & $4(10 \%)$ & $5(13 \%)$ & $30(77 \%)$ \\
\hline Q2.2 & 49 & $1(2 \%)$ & $2(4 \%)$ & $46(94 \%)$ \\
\hline Q2.3 & 14 & 0 & $1(7 \%)$ & $13(93 \%)$ \\
\hline Q3.1 & 5 & 0 & 0 & $5(100 \%)$ \\
\hline Q3.2 & 16 & 0 & 0 & $16(100 \%)$ \\
\hline Q3.3 & 2 & 0 & 0 & $2(100 \%)$ \\
\hline Q4.1 & 1 & 0 & 0 & $1(100 \%)$ \\
\hline $\mathrm{Q} 4.3$ & 2 & 0 & 0 & $2(100 \%)$ \\
\hline Total & 159 & $5(3 \%)$ & $10(6 \%)$ & $144(91 \%)$ \\
\hline
\end{tabular}

506

507 Table 5: Breakdown of the different types of 'Unclear' classifications. Only Journals with unclear

508 models are listed. Categorizations are mutually exclusive and exhaustive. Journals are referred by

509 the labels assigned in Table 1. 


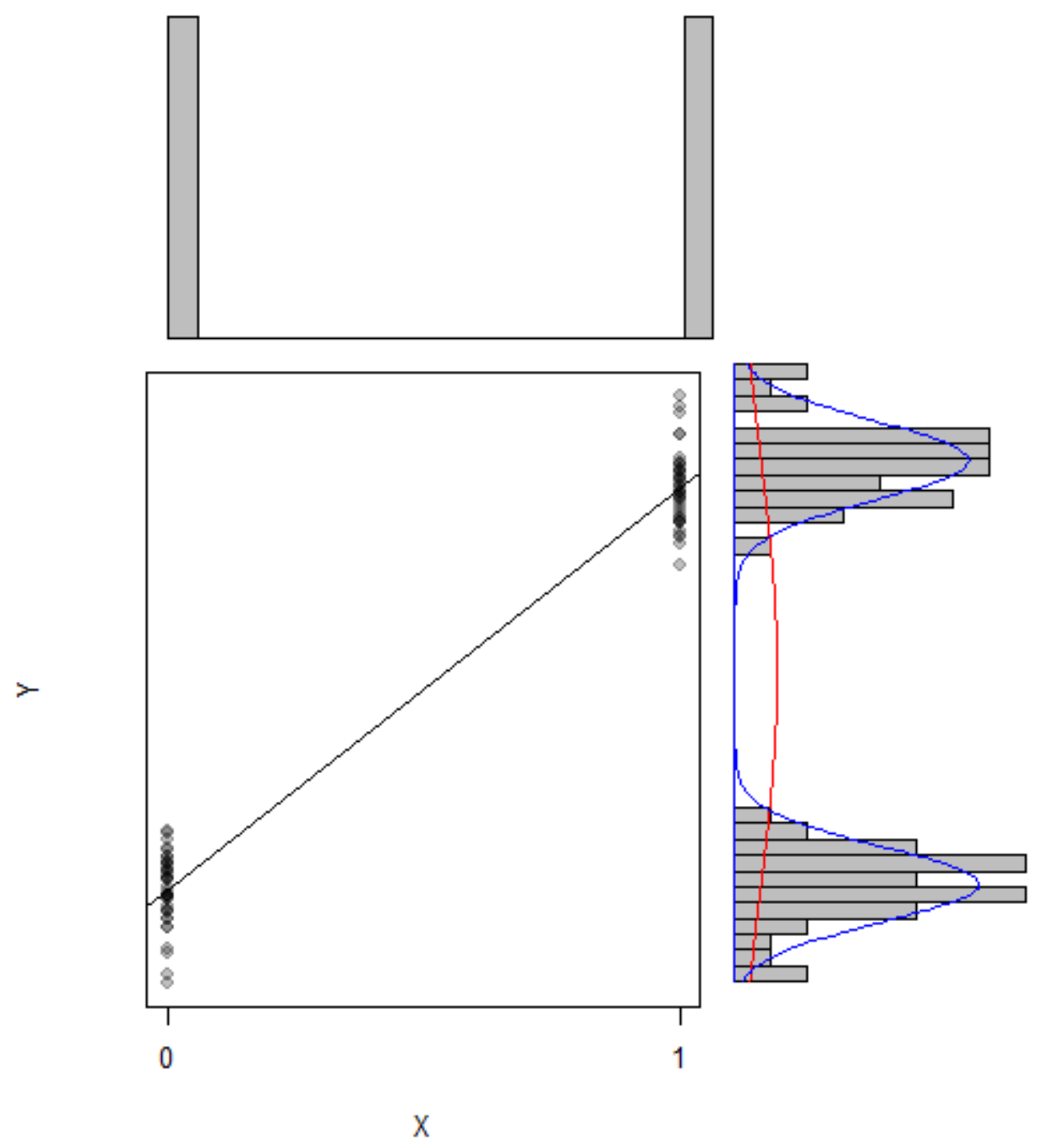

512

513 Figure 1: Simulated example of a $t$-test based on $n=40$ observations per group and no violations

514 of the assumptions. The main panel shows a scatterplot of $(X, Y)$-scores. The red curve

515 corresponds to the best-fitting normal distribution for $Y$, where the blue curves correspond to the

516 best-fitting normal distribution for both subpopulations of $Y$. The histograms in the top and side

517 panels clearly indicate non-normality for $X$ and $Y$. However, within both subpopulations the

518 distribution is normal 


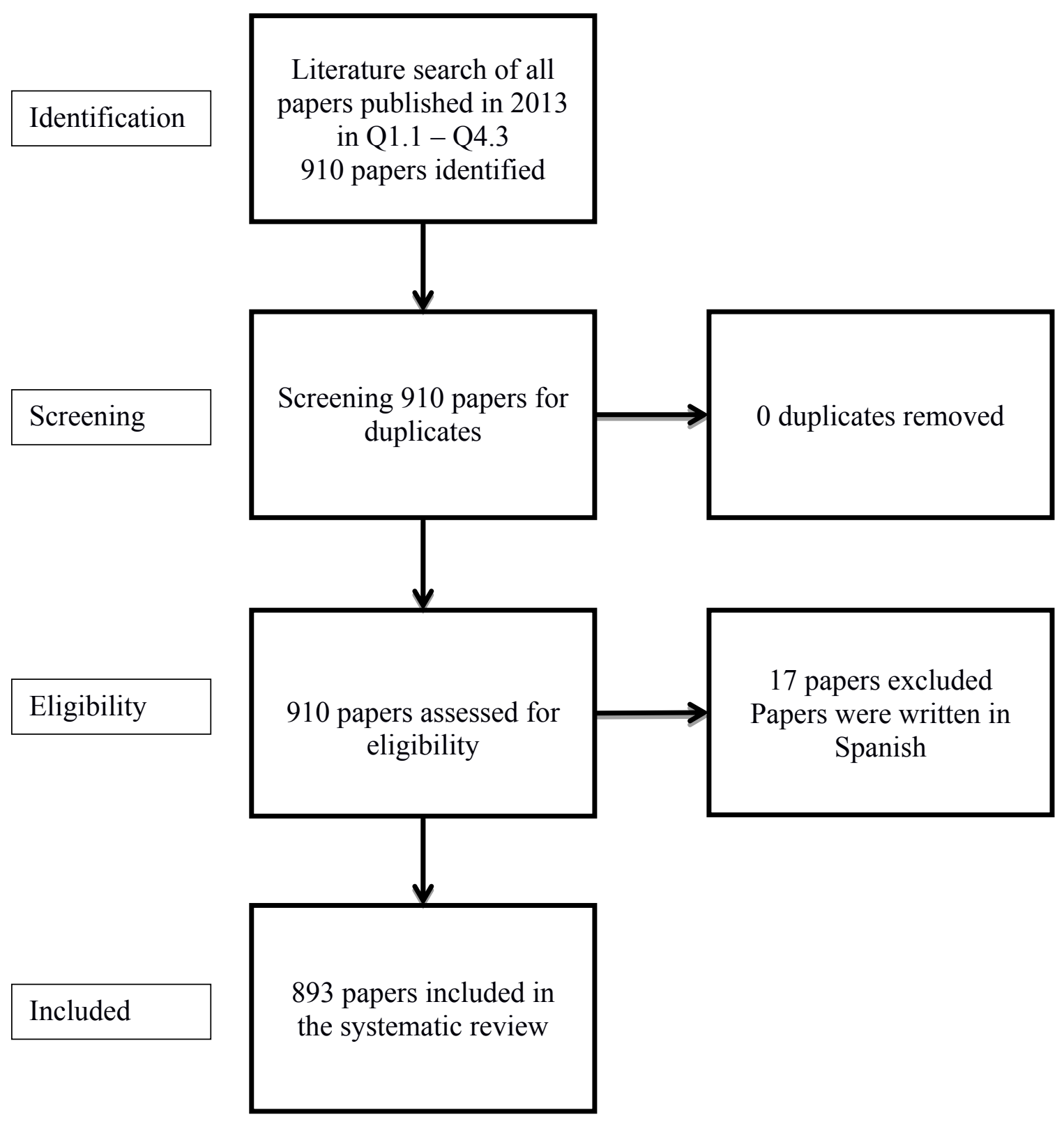

521 Figure 2: Prisma flow diagram of included records 